\title{
Editorial
}

\section{Liquid Crystal Research: Current Trends and Future Perspectives}

\author{
Durga P. Ojha, ${ }^{1}$ Hamit Yurtseven, ${ }^{2}$ Ashish K. Prajapati, ${ }^{3}$ and M. L. N. Madhu Mohan ${ }^{4}$ \\ ${ }^{1}$ School of Physics, Sambalpur University, Jyoti Vihar, Sambalpur, Odisha 768 019, India \\ ${ }^{2}$ Department of Physics, Middle East Technical University, 06531 Ankara, Turkey \\ ${ }^{3}$ Department of Applied Chemistry, Faculty of Technology and Engineering, The Maharaja Sayajirao University of Baroda, \\ Vadodara 390 001, India \\ ${ }^{4}$ Department of Electronics and Communications Engineering, Bannari Amman Institute of Technology, \\ Sathyamangalam 638 401, India \\ Correspondence should be addressed to Durga P. Ojha; durga_ojha@hotmail.com
}

Received 3 September 2013; Accepted 3 September 2013

Copyright (C) 2013 Durga P. Ojha et al. This is an open access article distributed under the Creative Commons Attribution License, which permits unrestricted use, distribution, and reproduction in any medium, provided the original work is properly cited.

Liquid crystals (LCs) are materials with unique properties mainly due to the presence of partially ordered phases. The ability to form ordered domains is involved in many applications of LCs in electronic devices such as liquid crystal displays (LCDs), spatial modulators, and TV and laptop screens. The occurrence of mesophases in these materials constitutes many challenging research problems. LCs are undergoing a scientific renaissance, both fundamentally and technologically, they have become a pervasive feature of everyday life.

The exploration of these molecular materials is still a challenge since the rapid development of display technology demands new LC materials, which possess as wide a range of properties as possible. The phase structures in these materials involve tricky problems. LCD technology has integrated itself into many facets of our daily lives. They have truly been instrumental in the progression and development of electronic devices. The development and application of materials with well-defined molecular and bulk properties are becoming increasingly important for contemporary technologies. The ability to simulate/synthesize new LC materials with properties and functions far beyond our current reach could lead to meso/nanoscale electronic and mechanical devices that far outstrip our current technological capabilities.

Due to their highly anisotropic constitution, LC materials are quite promising for applications in the field of molecular electronics and optoelectronics. Recently, keeping in pace with topical science, they have entered into the fascinating domains of space, nanoscience, and technology as well. Even though advances in synthesis and characterization explore many achievements in this regard, it is often desirable to have a sense of material behaviour before it is synthesized. The basic chemical constitution and building blocks of liquid crystal are much helpful, and they act as a model compound to deal with many problems in materials physics and chemistry.

This special issue is aimed at both basic and applied problems, concentrating on four major areas, that is, molecular design, simulation, synthesis, and characterization of liquid crystal materials. The articles in this special issue address some aspects of theoretical formalism and to validate application potentiality of LC compounds. Therefore, it is expected that the research output of the proposed special issue will magnetize a wide variety of research workers from crystallographers, molecular physicists, and biophysicists to structural chemists, organic chemists, polymer chemists, and drug industry, and so forth. This special issue brings together some of the latest developments in this field of liquid crystal research.

\section{Acknowledgments}

We would like to thank all the authors for their excellent contributions to this special issue and all the reviewers for 
their suggestions and decisive comments. We also expect that the special issue will stimulate the continuing efforts to intensify the liquid crystal research.

Durga P. Ojha

Hamit Yurtseven

Ashish K. Prajapati

M. L. N. Madhu Mohan 

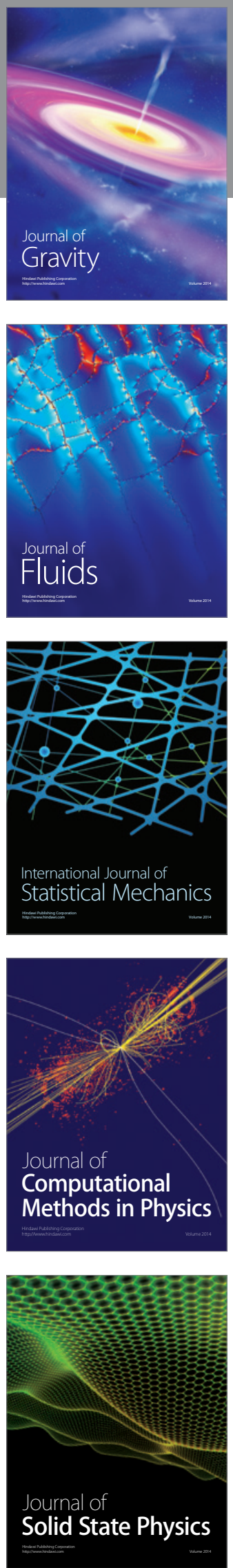

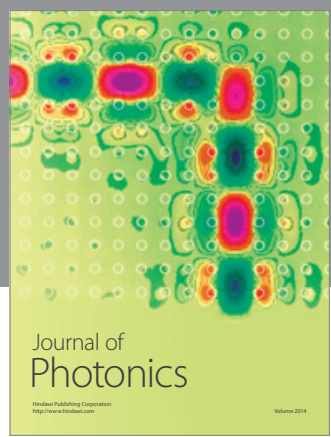

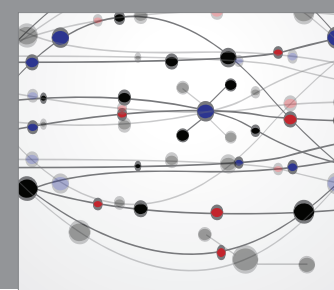

The Scientific World Journal

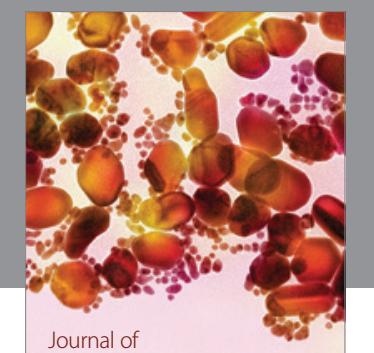

Soft Matter
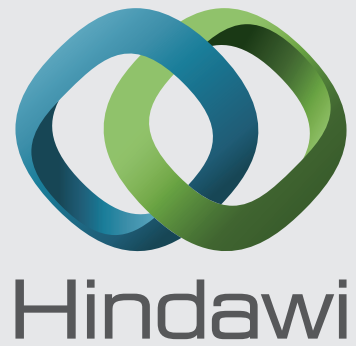

Submit your manuscripts at

http://www.hindawi.com
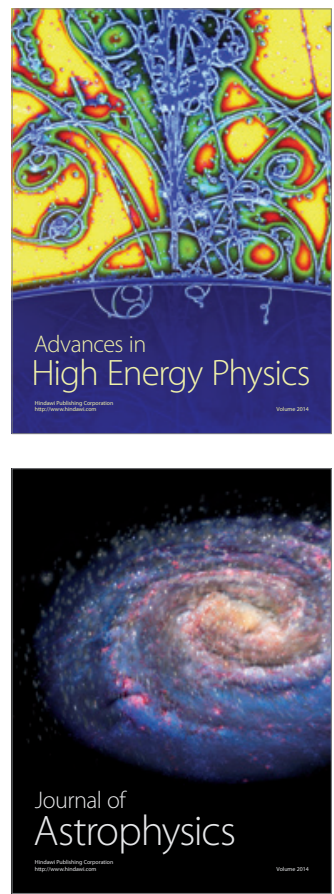
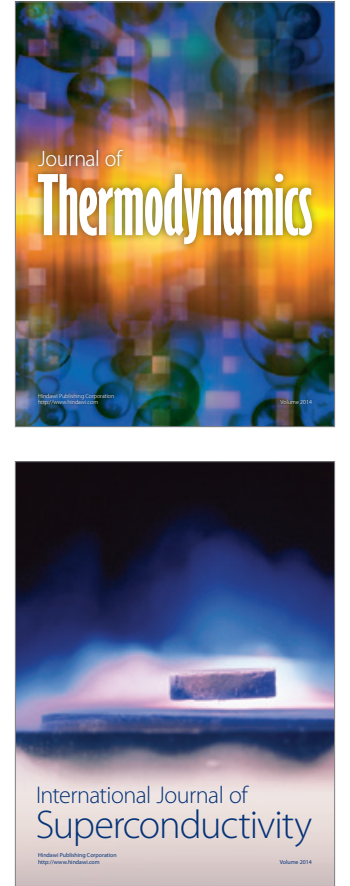
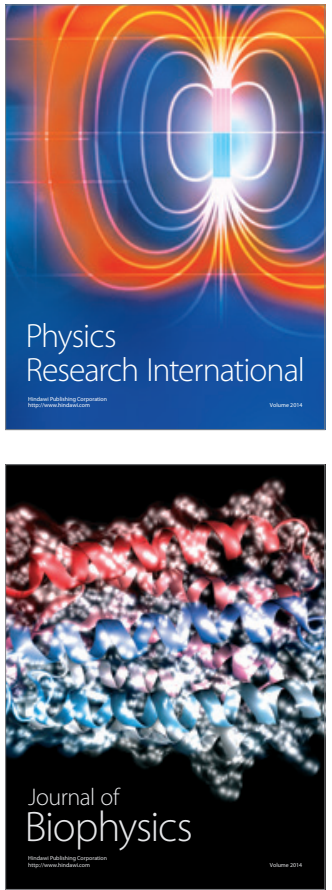
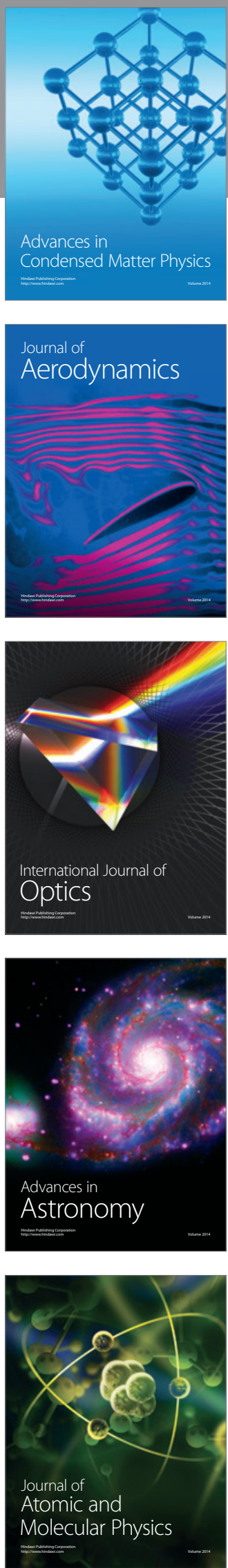